\title{
Crystal structure and functional implication of the RUN domain of human NESCA
}

\author{
Qifan Sun ${ }^{1,2}$, Chuanhui Han ${ }^{2,3}$, Lan Liu ${ }^{1,2}$, Yizhi Wang ${ }^{1,2}$, Hongyu Deng ${ }^{3}$, Lin Bai ${ }^{\circledR}$, Tao Jiang $^{1 凶}$ \\ ${ }^{1}$ National Laboratory of Biomacromolecules, Institute of Biophysics, Chinese Academy of Sciences, Beijing 100101, China \\ ${ }^{2}$ Graduate University of Chinese Academy of Sciences, Beijing 100039, China \\ ${ }^{3}$ Chinese Academy of Sciences Key Laboratory of Infection and Immunity, Institute of Biophysics, Chinese Academy of \\ Sciences, Beijing 100101, China \\ $\triangle$ Correspondence: tjiang@sun5.ibp.ac.cn (T. Jiang), bailin2002@moon.ibp.ac.cn (L. Bai) \\ Received May 24, 2012 Accepted July 9, 2012
}

\begin{abstract}
NESCA, a newly discovered signaling adapter protein in the NGF-pathway, contains a RUN domain at its $\mathrm{N}$-terminus. Here we report the crystal structure of the NESCA RUN domain determined at $2.0-\AA$ resolution. The overall fold of the NESCA RUN domain comprises nine helices, resembling the RUN domain of RPIPx and the RUN1 domain of Rab6IP1. However, compared to the other RUN domains, the RUN domain of NESCA has significantly different surface electrostatic distributions at the putative GTPase-interacting interface. We demonstrate that the RUN domain of NESCA can bind H-Ras, a downstream signaling molecule of TrkA, with high affinity. Moreover, NESCA RUN can directly interact with TrkA. These results provide new insights into how NESCA participates in the NGF-TrkA signaling pathway.
\end{abstract}

KEYWORDS NESCA, RUN domain, crystal structure, TrkA, Ras

\section{INTRODUCTION}

NGF (nerve growth factor) was the first discovered neurotrophin (Levi-Montalcini, 1987; Shooter, 2001), and plays an especially important role in regulating cellular interactions that control cell survival and differentiation (Jacobson, 1997). NGF interacts with two entirely distinct groups of receptors (Rodrigueztebar et al., 1990; Frade and Barde, 1998): p75 neurotrophin receptor (p75NTR), a low-affinity receptor that has a similar affinity to each of the neurotrophins; and TrkA, a member of the Trk family of receptor tyrosine kinases, which binds selectively to NGF (Reichardt, 2006). NGF binding activates the kinase domain of TrkA, leading to autophosphorylation (Gryz and Meakin, 2000). The resulting phosphotyrosines become docking sites for adaptor proteins involved in signal transduction pathways that lead to the activation of Ras, Rac, phosphatidylinositol 3-kinase (PI3-K), phospholipase C- $\mathrm{y}$ (PLC- $\mathrm{y}$ ) and other effectors (Kaplan and Miller, 2000; Huang and Reichardt, 2003). NESCA (new molecule containing an $\mathrm{SH} 3$ domain at the carboxyl terminus), a novel signaling adapter protein, was discovered to be involved in the NGF-TrkA-MEK pathway (MacDonald et al., 2004). NESCA mobilizes from the cytoplasm to the nuclear membrane in response to neurotrophin, which is stimulated in a MEK-dependent process, and enhances neurite outgrowth in response to NGF.

NESCA, which is primarily expressed in the brain, contains an N-terminal RUN domain, a WW domain, a leucine zipper, an $\mathrm{SH} 3$ domain at the carboxyl terminus and several distinct proline-rich regions (Matsuda et al., 2000) (Fig 1A). The RUN domain is an important structural determinant for the nuclear translocation of NESCA. Recent studies indicate that NESCA also plays a role in neuronal differentiation by regulating dendritic spine dynamics as an adaptor protein (Macdonald et al., 2012). This protein interacts with NEMO and TRAF6 through its $\mathrm{N}$-terminal region (RUN domain), serving as a novel molecular link between NGF signaling and the IKK complex activity (Napolitano et al., 2009).

Even though NESCA plays an important role in the cell signaling, there are no reports on its structure. To better understand the structural and functional characteristics of NESCA, we determined and analyzed the crystal structure of its N-terminal RUN domain (referred to NESCA-RUN in this paper). The overall fold of the NESCA RUN domain comprises nine helices, similar to other RUN domains with solved 
A

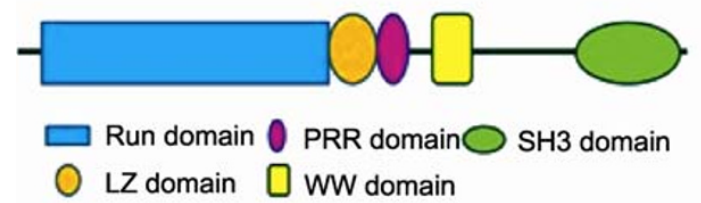

C

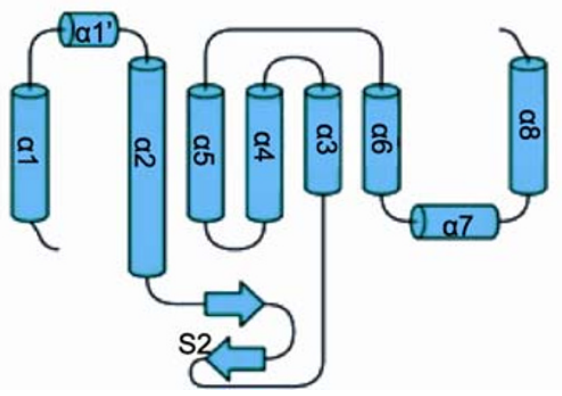

B

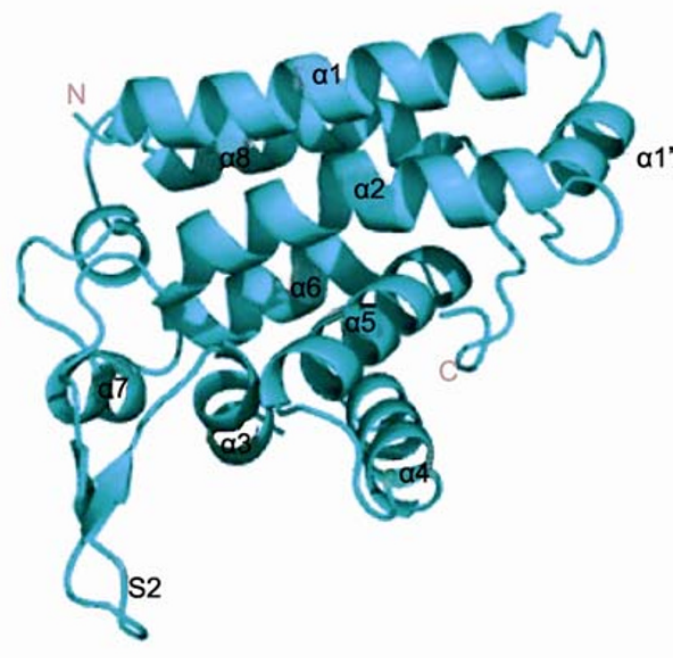

Figure 1. Structural overview of NESCA. (A) Schematic representation of NESCA. (B) Overall structure of the NESCA-RUN monomer, which contains $9 \alpha$-helixes and some linker peptides. (C) Topology model for NESCA-RUN.

structures. However, the putative GTPase-interacting interface of NESCA is different from that of other RUN domains. We demonstrated direct binding of NESCA-RUN with the small GTPase $\mathrm{H}$-Ras and the intracellular tyrosine kinase domain of TrkA by surface plasmon resonance (SPR). Further co-immunoprecipitation assays verified the interaction between NESCA-RUN and the intracellular tyrosine kinase domain of TrkA. These results provide new insight into the involvement of NESCA in the TrkA receptor mediated NGF pathway.

\section{RESULTS}

\section{Overall crystal structure of the RUN domain of NESCA}

We designed various constructs of the RUN domain of NESCA, but only the construct containing residues 8-198 was successfully crystallized. Two mutations (L150M and L152M) were introduced for solving the phase by the SeMet-based single wavelength anomalous diffraction (SAD) method. The crystal belongs to the space group $\mathrm{P} 2{ }_{1}$, with unit cell constants of $a=38.10 \AA, b=93.66 \AA, c=44.48 \AA$, and $\beta$ $=99.54^{\circ}$ (Table 1); and contains two identical monomers related by a non-crystallographic 2-fold axis per asymmetric unit (Fig. 2A).

Each monomer contains $9 \alpha$-helices, 1 short $\beta$ sheet and some linker peptides, the whole structure adopts a globular fold (Fig. 1B and 1C). $\alpha 1, \alpha 5$ and $\alpha 8$ are arranged in parallel, with $\alpha 2$ and $\alpha 6$ anti-parallel to them. The other 4 helices ( $\alpha 1$ ', $\alpha 3, \alpha 4$ and $\alpha 7$ ) are found at a nearly 90 -degree angle relative to the other five helices. We also determined the structure of the native construct by molecular replacement with the
Table 1 Data collection and refinement statistics

\begin{tabular}{ll}
\hline Space group & $P 2_{1}$ \\
\hline Cell parameters & $\begin{array}{l}a=39.11 \AA, b=94.20 \AA \\
c=44.60 \AA, \beta=99.54^{\circ}\end{array}$ \\
Resolution $(\AA)$ & $35.7-2.00(2.07-2.00)$ \\
Total reflections & 147,473 \\
Unique reflections & $20,352(2144)$ \\
Completeness $(\%)$ & $99.63(99.42)$ \\
Rmerge $(\%)^{\mathrm{a}}$ & $5.2(10.3)$ \\
l/s $(\mathrm{l})$ & $3.9(2.8)$ \\
R factors & \\
$\mathrm{R}_{\text {work }}(\%)^{\mathrm{b}}$ & 20.5 \\
$\mathrm{R}_{\text {free }}(\%)^{\mathrm{c}}$ & 21.3 \\
R.m.s.d. values & \\
Bond lengths $(\AA)$ & 0.007 \\
Bond angles $\left(^{\circ}\right)$ & 1.098 \\
B factor $\left(\AA^{2}\right)$ & \\
Main chain $\left(\AA^{2}\right)$ & 15.33 \\
Side chain and water $\left(\AA^{2}\right)$ & 17.14 \\
Ramachandran plot statistics & \\
Favored regions & $95.88 \%$ \\
Allowed regions & $4.12 \%$ \\
Outlier regions & 0 \\
\hline
\end{tabular}

${ }^{a} R_{\text {merge }}=\sum_{h k \mid} \sum_{i} I_{h k l, i}<\left|>_{h k l}\right| / \sum_{h k l} \sum_{i} I_{h k l,}$, where $I_{h k l}$ is the intensity of a reflection and $\langle\mid\rangle_{\mathrm{hkl}}$ is the average of the observations of this reflection and its symmetry equivalents.

${ }^{\mathrm{b}} \mathrm{R}_{\text {cryst }}=\sum\left\|\mathrm{F}_{\text {obs }}|-| \mathrm{F}_{\text {calc }}\right\| / \sum\left|\mathrm{F}_{\text {obs }}\right|$, where $\mathrm{F}_{\text {obs }}$ and $\mathrm{F}_{\text {calc }}$ are the observed and calculated structure factor amplitudes, respectively.

${ }^{c} R_{\text {free }}$ as $R_{\text {cryst }}$ but calculated with $10 \%$ of the data excluded from the refinement. 
A

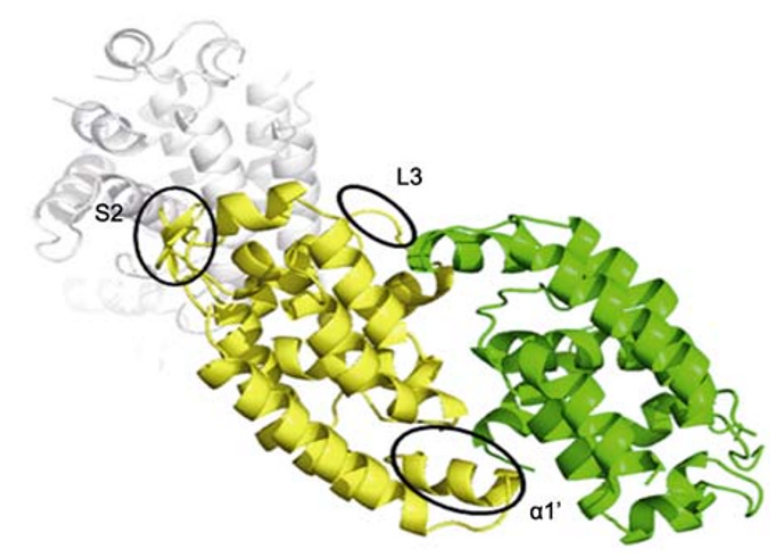

B

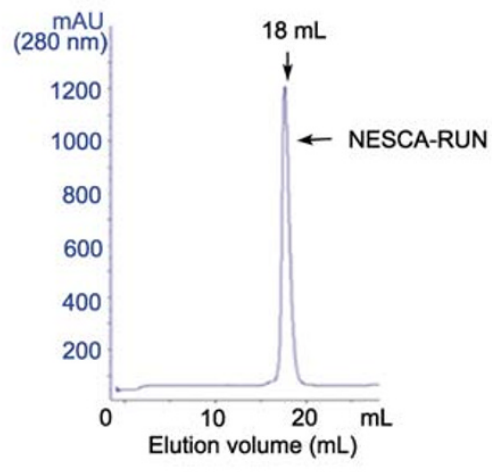

c

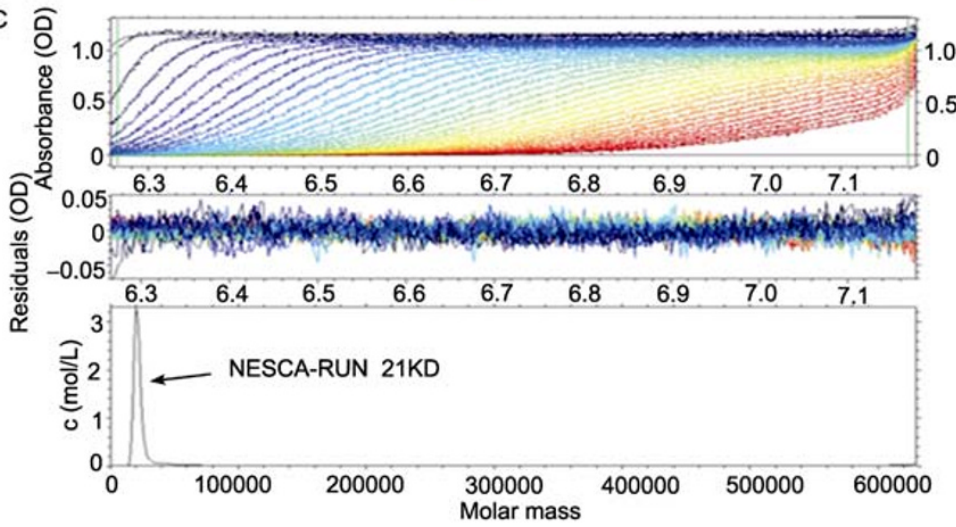

Figure 2. Monomer-monomer interaction of NESCA-RUN in the crystal and nonoligomeric behavior in solution. (A) Overall structure of the NESCA-RUN dimer. The two monomers are colored yellow and green, and the symmetry molecule is colored white. The regions responsible for the interaction between monomers and the symmetry molecule are circled. (B) Size-exclusion column chromatography of NESCA-RUN. (C) Sedimentation velocity experiments revealed that the molecular weight of the protein is about $21 \mathrm{kDa}$.

Se-Met derivative structure as a model. The wild-type structure adopts the same structure as the mutant (data not shown). Because the Se-Met derivative structure was solved at a higher resolution, it is used for further discussion.

The dimeric structure of NESCA-RUN is formed by the two monomers in the asymmetrical unit through hydrogen bonding and salt bridges as well as hydrophobic interactions between $\alpha 1^{\prime}$ and $\mathrm{L} 3$ of both monomers (Fig. 2A). The buried area of the two monomers is $712 \AA^{2}$, with each monomer contributing about $306 \AA^{2}$, corresponding to $12.5 \%$ of the total area of one monomer. Gel filtration chromatography showed that the NESCA-RUN construct used for crystallization tends to be monomeric (Fig. 2B). We further investigated its oligomeric state in solution using ultracentrifugation. These results indicated that the molecular weight of the protein is $\sim 21 \mathrm{kDa}$, corresponding to the molecular weight of a monomer (Fig. $2 \mathrm{C}$ ). In addition, there is no evidence concerning the biological relevance of NESCA-RUN dimerization. Therefore, it is likely that the dimeric assembly of NESCARUN was caused by crystal packing.

\section{Comparison between the structure of NESCA-RUN and other RUN domain structures}

The overall fold of NESCA-RUN is similar to that of the RUN domain of RPIPx (Kukimoto-Niino et al., 2006), with a root-mean-square deviation (rmsd) of $2.3 \AA$ over $182 \mathrm{Ca}$ atoms ( $23 \%$ sequence identity) (Fig. $3 \mathrm{~A}$ ), and the RUN1 domain of Rab6IP1 (Recacha et al., 2009), with an rmsd of 2.7 A over $182 \mathrm{Ca}$ atoms (23\% sequence identity) (Fig. 3B).

The main differences between NESCA-RUN and the other two RUN domains are localized to $\alpha 1^{\prime}$, the segment between $\alpha 2$ and $\alpha 3$ (S2), the $\alpha 3-\alpha 4$ loop (L3) and the $C$ terminus including $\alpha 8$. $\alpha 1$ ' of NESCA is a short helix located between $\alpha 1$ and $\alpha 2$, whereas the equivalent regions of RPIPx and Rab6IP1 are loops. Segment S2 of NESCA, which contains residues $72-89$ and folds into a two-stranded $\beta$ sheet, is much longer than that of both RPIPx and Rab6IP1 (Fig. $3 A-3 C$ ). The length of loop L3 of NESCA is slightly longer than that of RPIPx; but is much shorter than that of Rab6IP1, the majority of which is disordered. Notably, $\alpha 1$ ', S2, and L3 
A

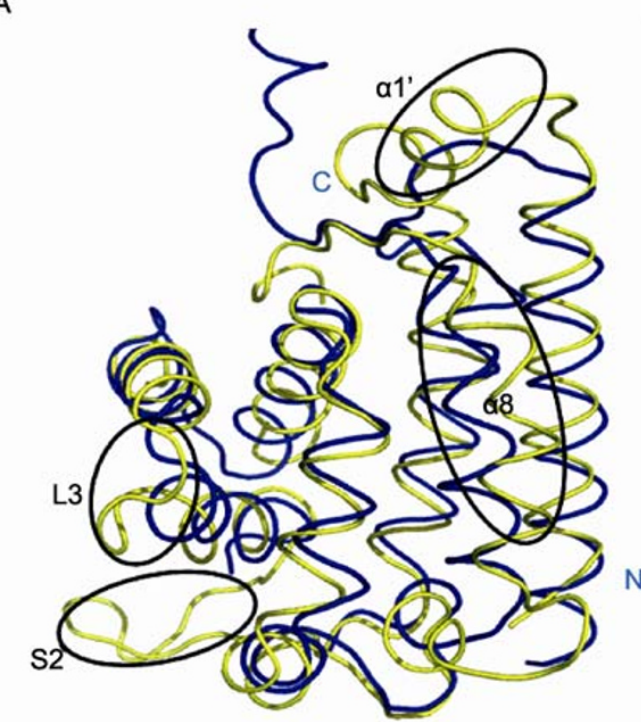

B

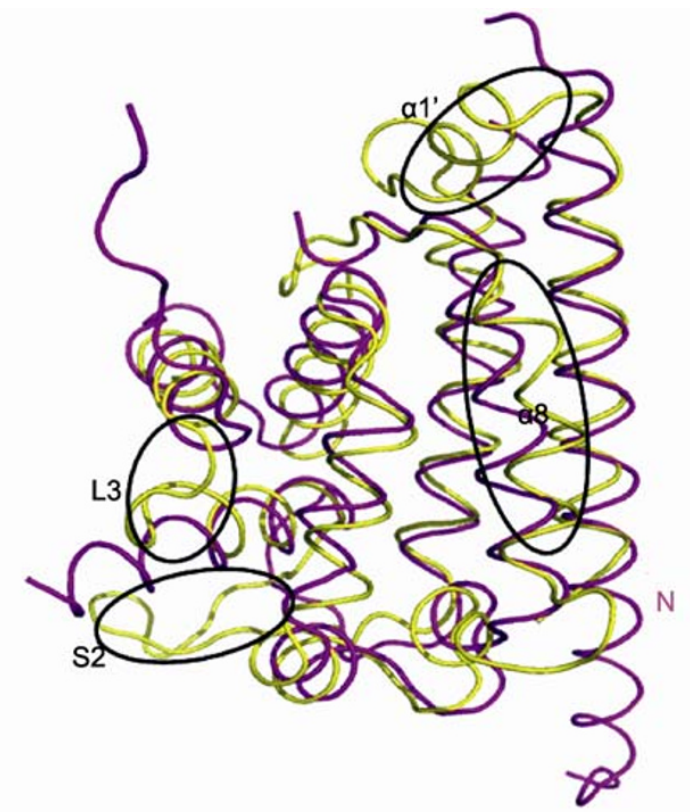

C

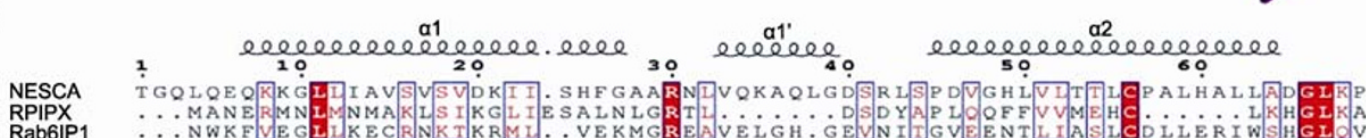

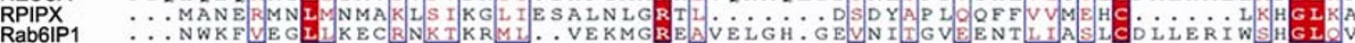

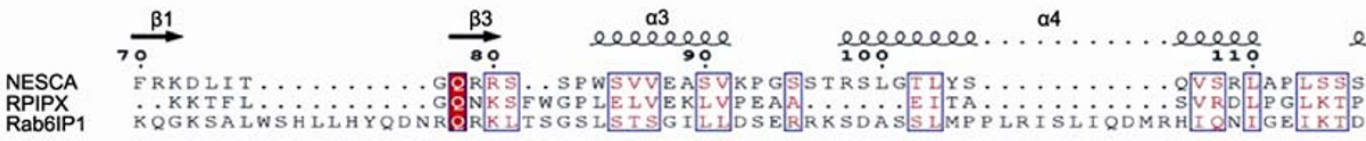

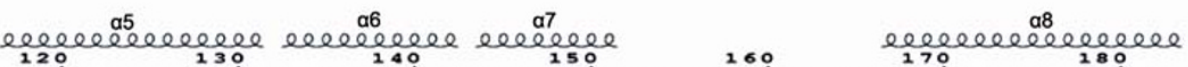

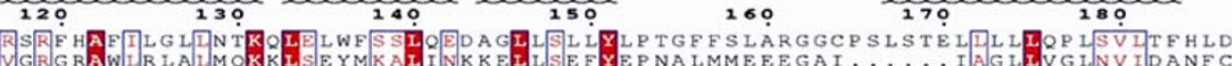

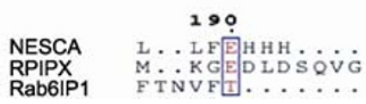

Figure 3. Structural comparison of RUN domains of NESCA, RPIPx and Rab6IP1. (A) Structural superimposition of NESCA-RUN (colored in yellow) with the RUN1 domain of RPIPX (colored in blue). Differences between the two proteins are found in $\alpha 1$ ', the segment between $\alpha 2$ and $\alpha 3$ (S2), the $\alpha 3-\alpha 4$ loop (L3) and $\alpha 8$, which are marked with black circles. (B) Structural superimposition of NESCA-RUN (colored in yellow) with the RUN1 domain of Rab6IP1 (colored in purple). Differences between these proteins are found in $\alpha 1$ ', S2, L3 and $\alpha 8$, which are marked with circles. (C) Sequence alignments of different RUN domains. This alignment was constructed using Kalign Multiple Sequence Alignments and ESPript2.2. The secondary structures for the NESCA RUN domain are given on top of the sequences. Primary sequences used in the alignment: mouse RPIPx (GI: 34849578), mouse Rab6IP1 (GI: 5869934) and human NESCA (GI: 5821424).

are all involved in interactions between the monomers (Fig. $3 \mathrm{~A}$ and $3 \mathrm{~B}$ ). As shown above by gel filtration chromatography and ultracentrifugation experiments, NESCA-RUN tends to act as a monomer in solution. Thus, the free $\alpha 1$ ', S2 and L3 regions may serve as binding sites for functionally related proteins. In addition, $\alpha 8$ of NESCA-RUN is different from that of RPIPx and Rab6IP1.

\section{Interaction between NESCA-RUN and H-Ras}

It has been suggested that the RUN domain binds small GTPase proteins because the RUN domain is usually found in proteins that regulate small GTPase activity in mammals (Janoueix-Lerosey et al., 1998; Callebaut et al., 2001). Experimental evidence suggests that NESCA plays an impor- 
tant role in the NGF signaling pathway (MacDonald et al., 2004). As a member of the small GTPase family, Ras also functions as one of the downstream signaling proteins in the TrkA pathway (Campbell et al., 1998; Grewal et al., 1999). Thus, we investigated whether the NESCA RUN domain can interact with Ras. We performed a SPR experiment to measure the binding affinities between NESCA-RUN and human $\mathrm{H}-\mathrm{Ras}$, an isoform of Ras. Our results indicated that $\mathrm{H}$-Ras exhibits a strong binding affinity to the NESCA-RUN domain, with a $\mathrm{K}_{\mathrm{d}}$ of $6.68 \times 10^{-8} \mathrm{~mol} / \mathrm{L}$ (Fig. $4 \mathrm{~A}$ ).

In the crystal structure of the RUN domain of Rab6IP1 in complex with Rab6a (GTP), Rab6 binding is mediated by noncontiguous $\alpha 1$ and $\alpha 8$ of the RUN domain. As the structure of NESCA-RUN shows high similarity with that of Rab6IP1, we wondered whether the interaction between NESCA-RUN and Ras is similar to that between Rab6IP1 and Rab6a. However, sequence alignment showed that the residues of NESCA that correspond to the Rab6 interacting residues in Rab6IP1 are not conserved (Fig. $3 \mathrm{C}$ ). Moreover, the electrostatic distributions of this interface in these two RUN domains showed opposite electrostatic potentials (Fig. 5). The electrostatic distributions of the Rab6a-interacting interface $(\alpha 1, \alpha 8)$ of the RUN1 domain of Rab6IP1 are similar to those of RPIPx, with positive charge in the middle and negative charge on both sides. In contrast, the electrostatic distributions of $\alpha 1$ and $\alpha 8$ of NESCA-RUN show negative charge in the middle and positive charge on both sides. Differences between $a 8$ of NESCA-RUN and that of RPIPx and Rab6IP1 further indicate that NESCA-RUN has distinct properties from the other two RUN domains. Furthermore, considering that NESCA-RUN has some characteristic monomer-monomer interaction regions ( $\alpha 1$ ', S2 and L3) (Fig. 2), we posit that the interaction between NESCA-RUN and $\mathrm{H}$-Ras may be different from that between Rab6IP1 and Rab6a. This speculation was supported by our docking calculation using program ZDOCK (Pierce et al., 2011), which showed that the H-Ras binding region of NESCA-RUN were located at S2 and L3 domain (Fig. 6A), whereas in the

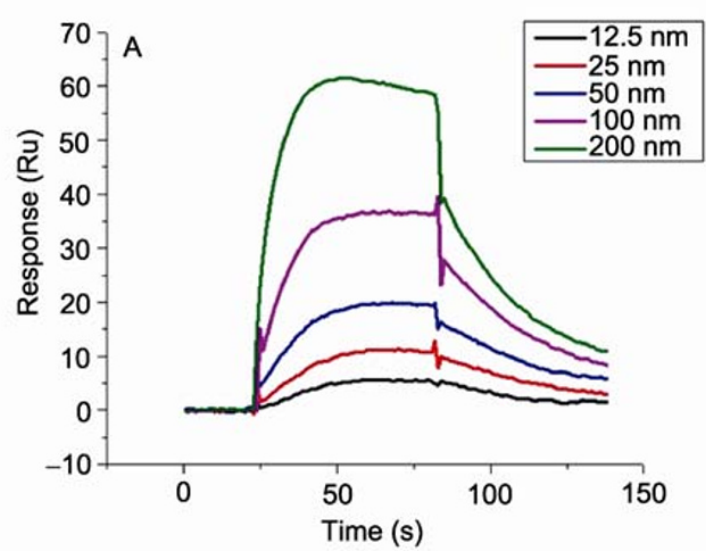

C

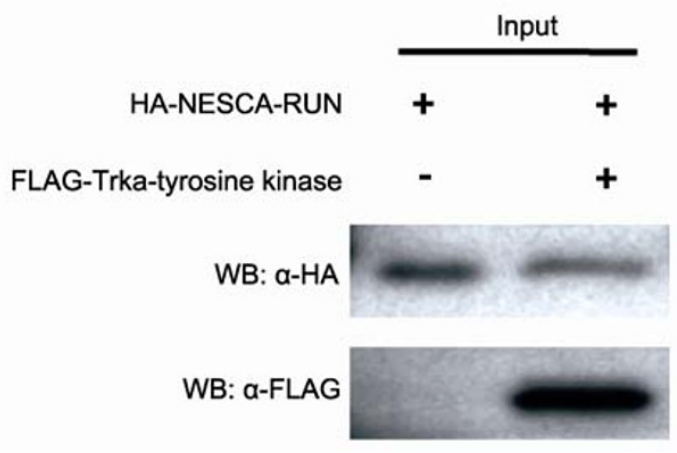

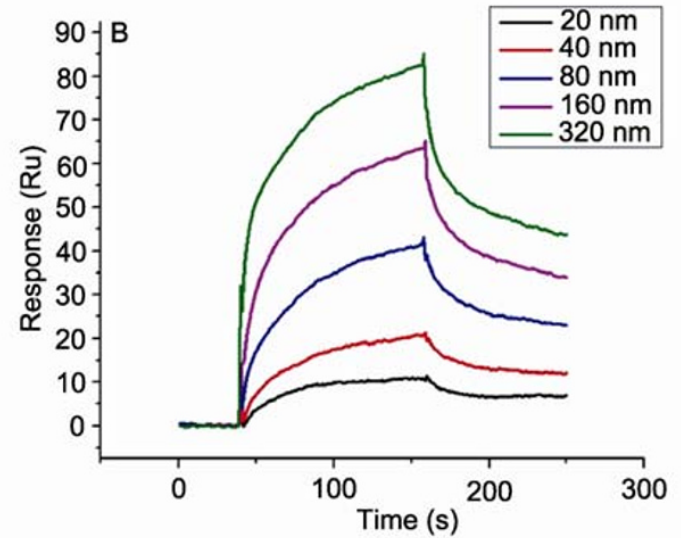

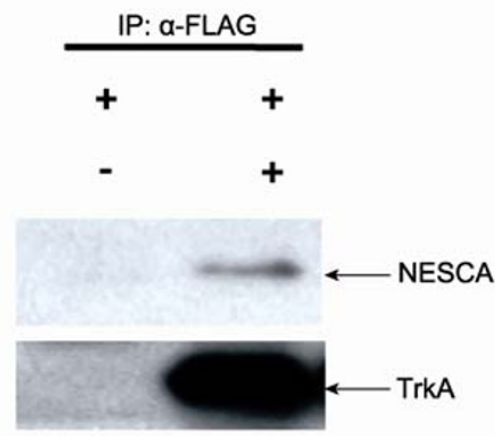

Figure 4. Interaction analysis by BIAcore and Co-IP assays. (A) Binding assays measuring the interaction of the NESCA-RUN domain and Ras protein. Representative sensorgrams were obtained from injections of Ras at concentrations of 12.5, 50, 100, and $200 \mathrm{nmol} / \mathrm{L}$. (B) Binding assays measuring the interaction of the NESCA-RUN domain and the TrkA tyrosine kinase domain. Representative sensorgrams were obtained from injections of the TrkA tyrosine kinase domain at concentrations of 20, 40, 80, 160, 320 and $640 \mathrm{nmol} / \mathrm{L}$. (C) Interaction between NESCA-RUN domain and TrkA tyrosine kinase domain analysised by Co-IP method. 293T cells were transfected with pHA-NESCA-RUN and pTAG-TrkA tyrosine kinase domain or pHA-NESCA-RUN alone. At $48 \mathrm{~h}$ post-transfection, the cells were lysed and subjected to Co-IP and Western blot analysis. 
A

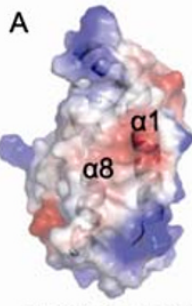

NESCA-RUN

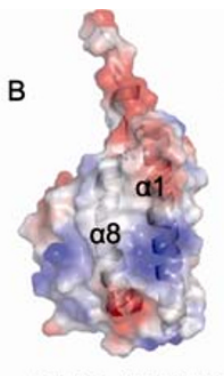

RUN of PRIPX

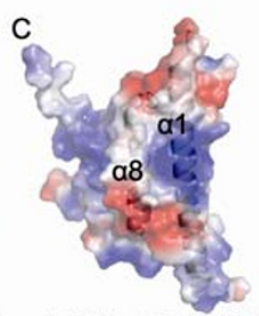

RUN1 of Rab6IP1

Figure 5. The electrostatic distributions of the RUN domains of NESCA, RPIPx and Rab6IP1. (A) The electrostatic distributions of $\alpha 1$ and $\alpha 8$ of the RUN domain of NESCA. (B) The electrostatic distributions of $\alpha 1$ and $\alpha 8$ of the RUN domain of RPIPx. (C) The electrostatic distributions of the Rab6a-interacting interface $(\alpha 1, \alpha 8)$ of the RUN1 domain of Rab6IP1.

Rab6a-Rab6IP1 complex, $\alpha 1$ and $\alpha 8$ of the RUN1 domain of Rab6IP1 are interact with Rab6a (Fig. 6B).

\section{Interaction between NESCA-RUN and TrkA}

Previous work showed that NESCA mobilizes from the cytoplasm to the nuclear envelope and facilitates neurite outgrowth in response to neurotrophin stimulation (MacDonald et al., 2004). Moreover, a yeast two-hybrid screen experiment revealed an interaction between the intracellular domain of TrkA and NESCA. However, it remained unclear whether these two proteins interact with each other directly and which region of NESCA is involved in the interaction. We hypothe-

A

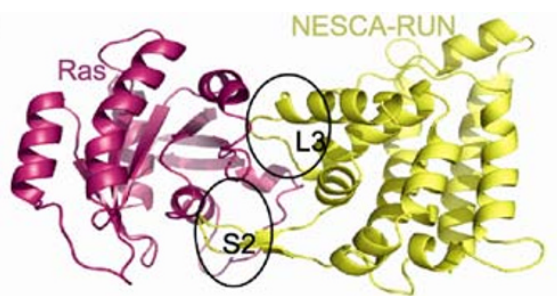

B

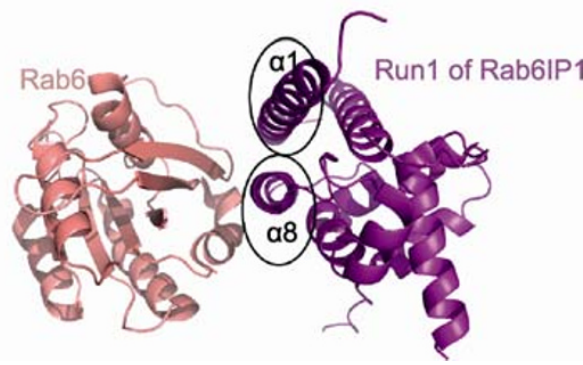

Figure 6. RUN-GTPase complex models. (A) The docking result of NESCA-RUN (colored in yellow) and Ras (colored in warm pink) by using ZDOCK, the interaction regions lie in L2 and S3, which was marked with black circles. (B) The crystal structure of Rab6a-RabIP1 complex (Rab6IP1-RUN1 is colored in purple and Rab6a is colored in deep salmon), the interaction regions locate at $\alpha 1$ and $\alpha 8$, which was marked with black circles.

sized the direct binding occurs between NESCA and TrkA because previous work demonstrated a direct interaction between Etk, a tyrosine kinase, and a RUN domain-containing protein RUFY1 (Yang et al., 2002).

To test this hypothesis, we used SPR to measure the binding affinity between NESCA-RUN and the tyrosine kinase domain of the TrkA receptor. Our results indicated that NESCARUN interacts directly with the tyrosine kinase domain of the TrkA receptor in a strong binding affinity $\left(K_{d}\right.$ of $\left.4.25 \times 10^{-8} \mathrm{~mol} / \mathrm{L}\right)$ (Fig. 4B). Co-immunoprecipitation experiments in HEK293T cells further confirmed the interaction between HA-NESCARUN and FLAG-TrkA tyrosine kinase domain (Fig. 4C).

\section{DISCUSSION}

We resolved the crystal structures of the RUN domain of NESCA at the resolution of $2.0 \AA$, and compared it with the RUN domain of RPIPX and Rab6IP1, which both are small GTPase interacting proteins. Using SPR assays and Co-IP experiments, we revealed that NESCA can bind to both TrkA and $\mathrm{H}$-Ras by its RUN domain. All these findings provide new insights into how NESCA was involved in the NGF pathway medicated by TrkA receptor.

It has been shown that NGF binding to the extracellular domain of TrkA will result in the activation of Ras, which can further activate MAPKs (Grewal et al., 1999; Ballif and Blenis, 2001). Previous work showed that this action occurs in response to phosphorylation at Y490 of TrkA. Phosphorylated Y490 provides a recruitment site for binding of the PTB domain of the adaptor protein Shc. After its own phosphorylation, Shc recruits the adaptor protein Grb2 and SOS, an exchange factor for Ras (and Rac) (Nimnual et al., 1998). Next, SOS activates nearby Ras protein. The presence of activated Ras then stimulates signaling through several downstream pathways, including those mediated by Class I PI3-kinases, Raf, and p38MAP kinase (Xing et al., 1998; Vanhaesebroeck et al., 2001). Our observation that NESCA can bind to both TrkA and Ras suggests that TrkA can recruit Ras by NESCA to nearby where Ras can be activated by SOS. This enrichment of Ras may increase the efficiency of the signaling transduction from TrkA to Ras, although a detailed mechanism awaits further investigation.

\section{MATERIALS AND METHODS}

\section{Cloning, expression and purification}

The fragment encoding residues 8-198 of human NESCA, which was purchased from Origene, was cloned into a pET-24a (+) vector with a His-tag at its C-terminus and transformed into the E. coli BL21 (DE3) strain. Cells from a freshly transformed clone were grown at $37^{\circ} \mathrm{C}$ in LB medium in the presence of $25 \mu \mathrm{g} / \mathrm{mL}$ kanamycin. When the OD at $600 \mathrm{~nm}$ reached approximately 0.6 , IPTG was added to a final concentration of $0.3 \mathrm{mmol} / \mathrm{L}$, and the incubation was continued at $16^{\circ} \mathrm{C}$ 
for $20 \mathrm{~h}$. The harvested cell pellets were resuspended in lysis buffer $(50 \mathrm{mmol} / \mathrm{L}$ Tris $\mathrm{pH} 8.0,500 \mathrm{mmol} / \mathrm{L} \mathrm{NaCl}, 5 \%$ glycerol $(\mathrm{v} / \mathrm{v}), 0.1$ $\mathrm{mmol} / \mathrm{L} \mathrm{PMSF}$, and $20 \mathrm{mmol} / \mathrm{L}$ imidazole) and sonicated. The fusion protein was purified on a Ni-NTA column followed by ion exchange chromatography using a 1-mL Resource S column (GE Healthcare). Gel filtration was performed as the final purification step, using a Superdex 200 10/300 GL column (GE Healthcare) in a buffer containing $50 \mathrm{mmol} / \mathrm{L}$ HEPES (pH 7.0), $150 \mathrm{mmol} / \mathrm{L} \mathrm{NaCl}, 2 \mathrm{mmol} / \mathrm{L}$ DTT, and $1 \mathrm{mmol} / \mathrm{L}$ EDTA. The protein was concentrated to $3 \mathrm{mg} / \mathrm{mL}$ for crystallization.

As there were no methionine residues in the truncated construct (8-198) of NESCA, we introduced two mutations (L150M and L152M), so the phase could be solved by the SeMet-based single wavelength anomalous diffraction (SAD) method. SeMet-substituted NESCA was overexpressed as previously reported (Doublie, 2007) and purified as described above.

The DNA fragment corresponding to the tyrosine kinase domain of human TrkA (residues 489-796) was cloned into a pFastBac1 vector. The protein was expressed in Sf9 insect cells and purified by affinity chromatography and gel filtration. Recombinant baculovirus was produced and amplified using Sf9 cells in serum-containing Trichoplusia ni medium-Fred Hink (TNM-FH) insect media at $27^{\circ} \mathrm{C}$. The Sf9 cells were cultivated in flasks in $2.5 \mathrm{~L}$ serum-free HyQ SFX medium (HyClone) at $27^{\circ} \mathrm{C}$. When the cell density reached $5 \times 10^{6}$ cells per milliliter, the cells were centrifuged and resuspended in $2.5 \mathrm{~L}$ fresh serum-free media and were infected with recombinant virus at an $\mathrm{MOI}$ of $>5$. The supernatant of the cultures was collected $72 \mathrm{~h}$ after infection. The expressed protein was purified by metal affinity chromatography on chelatin resin and size-exclusion chromatography on a Superdex 200 10/300 GL column (GE Healthcare).

\section{Crystallization}

Crystals of the NESCA-RUN domain were grown at $16^{\circ} \mathrm{C}$ by the hanging drop method, by mixing $1 \mu \mathrm{L}$ of protein solution with $1 \mu \mathrm{L}$ of reservoir solution $\mathrm{A}$, which was composed of $20 \%$ PEG3350 (w/ $/ \mathrm{V})$ and $0.2 \mathrm{~mol} / \mathrm{L} \mathrm{K}_{2} \mathrm{HPO}_{4}$. Single crystals were obtained by microseeding (Zhu et al., 2005). The SeMet-NESCA single crystals were grown under the same conditions.

\section{Data collection and structure determination}

The SeMet-NESCA-RUN diffraction data were collected at beamline $17 \mathrm{U}$ (BL17U) at the Shanghai Synchrotron Radiation Facility (SSRF) and were processed with HKL2000 (Otwinowski and Minor, 1997). The initial phases were obtained using the single-wavelength anomalous dispersion method and the program PHENIX (Adams et al., 2002). Coot was used to manually adjust and rebuild the models (Emsley and Cowtan, 2004). Further model refinement was carried out using Refmac5 (Murshudov et al., 1997) in the CCP4 suite (Adams et al., 2002) and PHENIX until acceptable $R_{\text {work }}(20.5 \%)$ and $R_{\text {free }}$ $(21.3 \%)$ values were obtained.

\section{Analytical ultracentrifugation}

The analytical ultracentrifugation experiments were carried out at $20^{\circ} \mathrm{C}$ with a Beckman XL-I analytical ultracentrifuge. The NESCA-RUN domain protein sample was diluted with buffer $(20 \mathrm{mmol} / \mathrm{L}$ HEPES, $150 \mathrm{mmol} / \mathrm{L} \mathrm{NaCl}, \mathrm{pH}$ 7.0) to a suitable concentration (when the absorbance at $280 \mathrm{~nm}$ reached 0.8 ). The interference sedimentation coefficient distribution was then calculated from the sedimentation velocity data using SEDFIT.

\section{Protein-protein docking}

For protein-protein interaction ZDOCK was used (Pierce et al., 2011). ZDOCK is a Fast Fourier Transform based protein docking program. It takes two PDB files and outputs the predicted structure of their complex. ZDOCK is a rigid body search with an initial stage algorithm, followed by RDOCK, an interface refinement minimization algorithm.

The protein receptor and protein ligand are treated as rigid bodies and all six rotational and translational degrees of freedom are fully explored. No contact information was used, which can filter hits or block residues during the search. Here we give input NESCA-RUN as receptor and $\mathrm{H}$-Ras (PDB 1BKD) as ligand. After running this server it's perform docking between these proteins and generate complex structures of NESCA-RUN and H-Ras.

\section{SPR experiments}

NESCA-RUN and the tyrosine kinase domain of human TrkA (residues 489-796) were expressed and purified as described above. The $\mathrm{H}$-Ras protein (human HRAS-6xhis fusion protein, residues 1-170) was purchased from ProteinTech Group. The interactions between NESCA-RUN and H-Ras and the TrkA tyrosine kinase domain were analyzed with a BIAcore 3000 instrument.

NESCA-RUN was immobilized on a CM5 chip (GE Healthcare) using an amine coupling kit, and the remaining coupling sites were blocked with $1 \mathrm{~mol} / \mathrm{L}$ ethanolamine $(\mathrm{pH} 8.5)$ as control. Ras and the TrkA tyrosine kinase domain were injected for $60 \mathrm{~s}$ at a flow rate of 10 $\mu \mathrm{L} / \mathrm{min}$ at various concentrations. All data were analyzed using $\mathrm{Bl}-$ Aevaluation 4.1 software to obtain corrected sensor-grams. The dissociation constants were calculated by fitting the data to a steady-state affinity model.

\section{Co-immunoprecipitation assay}

The DNA fragments corresponding to NESCA-RUN (residues 8-198) and TrkA tyrosine kinase domain (residues 489-796) were cloned into p3XFLAG-CMV-14 and pCMV-HA vectors, respectively. Plasmids were transfected into HEK293T cells using PEI transfection reagent. At $48 \mathrm{~h}$ posttransfection, cells were harvested and lysed for $30 \mathrm{~min}$ in cold lysis buffer $(50 \mathrm{mmol} / \mathrm{L}$ Tris- $\mathrm{HCl} \mathrm{pH} \mathrm{7.8,} 150 \mathrm{mmol} / \mathrm{L}$ $\mathrm{NaCl}, 5 \mathrm{mmol} / \mathrm{L}$ EDTA, $0.5 \%$ Triton X-100, 0.5\% NP-40, $0.1 \%$ deoxycholic acid, $1 \mathrm{mmol} / \mathrm{L} \mathrm{PMSF}$, and $1 \times$ protease inhibitor cocktail). The cell lysates were centrifuged at $12,000 \mathrm{~g}$ for $15 \mathrm{~min}$, and then, the supernatants were incubated with Anti-FLAGM2 Affinity Gel (Sigma) at $4^{\circ} \mathrm{C}$ for $4 \mathrm{~h}$. The beads were then washed three times for $5 \mathrm{~min}$ each with lysis buffer, and bound proteins were eluted with $2 \times$ protein SDS PAGE loading buffer. The samples were then analyzed by Western blottings. 


\section{ACKNOWLEDGMENTS}

We would like to thank the staff at the Shanghai Synchrotron Radiation Facility (SSRF) for their assistance in data collection. We would also like to thank Y. Y. Chen for performing the Biacore analysis. This research was supported financially by the National Natural Science Foundation of China (Grant Nos. 31021062 and 31025009), and the National Basic Research Program (973 Program) (No. 2011CB910302).

\section{ABBREVIATIONS}

NESCA-RUN, NESCA run domain; NGF, nerve growth factor; NTR, neurotrophin receptor; PI3-K, phosphatidylinositol 3-kinase; PLC- $\gamma$, phospholipase C- $\gamma$; Rab6IP1, Rab6-interacting protein 1; Ras, rat sarcoma; RPIPx, Rap2-interacting protein $x$; SAD, SeMet-based single wavelength anomalous diffraction; SPR, surface plasmon resonance; TrkA, tyrosine-receptor kinase $A$

\section{REFERENCES}

Adams, P.D., Grosse-Kunstleve, R.W., Hung, L.W., loerger, T.R., McCoy, A.J., Moriarty, N.W., Read, R.J., Sacchettini, J.C., Sauter, N.K., and Terwilliger, T.C. (2002). PHENIX: building new software for automated crystallographic structure determination. Acta Cryst D Biol Crystallogr 58, 1948-1954.

Ballif, B.A., and Blenis, J. (2001). Molecular mechanisms mediating mammalian mitogen-activated protein kinase (MAPK) kinase (MEK)-MAPK cell survival signals. Cell Growth Differ 12, 397-408.

Callebaut, I., de Gunzburg, J., Goud, B., and Mornon, J.P. (2001). RUN domains: a new family of domains involved in Ras-like GTPase signaling. Trends Biochem Sci 26, 79-83.

Campbell, S.L., Khosravi-Far, R., Rossman, K.L., Clark, G.J., and Der, C.J. (1998). Increasing complexity of Ras signaling. Oncogene 17, 1395-1413.

Doublie, S. (2007). Production of selenomethionyl proteins in prokaryotic and eukaryotic expression systems. Methods Mol Biol (Clifton, N.J.) 363, 91-108.

Emsley, P., and Cowtan, K. (2004). Coot: model-building tools for molecular graphics. Acta Cryst D Biol Crystallogr 60, 2126-2132.

Frade, J.M., and Barde, Y.A. (1998). Nerve growth factor: two receptors, multiple functions. Bioessays 20, 137-145.

Grewal, S.S., York, R.D., and Stork, P.J. (1999). Extracellular-signal-regulated kinase signalling in neurons. Curr Opin Neurobiol 9, 544-553.

Gryz, E.A., and Meakin, S.O. (2000). Acidic substitution of the activation loop tyrosines in TrkA supports nerve growth factor-independent cell survival and neuronal differentiation. Oncogene 19, 417-430.

Huang, E.J., and Reichardt, L.F. (2003). Trk receptors: Roles in neuronal signal transduction. Annu Rev Biochem 72, 609-642.

Jacobson, M.S. (1997). Adolescent nutritional disorders: prevention and treatment (New York, N.Y., New York Academy of Sciences).

Janoueix-Lerosey, I., Pasheva, E., de Tand, M.F., Tavitian, A., and de Gunzburg, J. (1998). Identification of a specific effector of the small GTP-binding protein Rap2. Eur J Biochem 252, 290-298.

Kaplan, D.R., and Miller, F.D. (2000). Neurotrophin signal transduction in the nervous system. Curr Opin Neurobiol 10, 381-391.

Kukimoto-Niino, M., Takagi, T., Akasaka, R., Murayama, K., Uchikubo-Kamo, T., Terada, T., Inoue, M., Watanabe, S., Tanaka, A., Hayashizaki, Y., et al. (2006). Crystal structure of the RUN domain of the RAP2-interacting protein x. J Biol Chem 281, 31843-31853.

Levi-Montalcini, R. (1987). The nerve growth factor 35 years later. Science 237, 1154-1162.

Macdonald, J.I., Dietrich, A., Gamble, S., Hryciw, T., Grant, R.I., and Meakin, S.O. (2012). Nesca, a novel neuronal adapter protein, links the molecular motor kinesin with the pre-synaptic membrane protein, syntaxin-1, in hippocampal neurons. J Neurochem. (In Press).

MacDonald, J.I.S., Kubu, C.J., and Meakin, S.O. (2004). Nesca, a novel adapter, translocates to the nuclear envelope and regulates neurotrophin-induced neurite outgrowth. J Cell Biol 164, 851-862.

Matsuda, S., Miyazaki, K., Ichigotani, Y., Kurata, H., Takenouchi, Y., Yamamoto, T., Nimura, Y., Irimura, T., Nakatsugawa, S., and Hamaguchi, M. (2000). Molecular cloning and characterization of a novel human gene (NESCA) which encodes a putative adapter protein containing SH3. Biochim Biophys Acta 1491, 321-326.

Murshudov, G.N., Vagin, A.A., and Dodson, E.J. (1997). Refinement of macromolecular structures by the maximum-likelihood method. Acta Cryst D Biol Crystallogr 53, 240-255.

Napolitano, G., Mirra, S., Monfregola, J., Lavorgna, A., Leonardi, A., and Ursini, M.V. (2009). NESCA: A New NEMO/IKKgamma and TRAF6 Interacting Protein. J Cell Physiol 220, 410-417.

Nimnual, A.S., Yatsula, B.A., and Bar-Sagi, D. (1998). Coupling of Ras and Rac guanosine triphosphatases through the Ras exchanger Sos. Science 279, 560-563.

Otwinowski, Z., and Minor, W. (1997). Processing of X-ray diffraction data collected in oscillation mode. Method Enzymol 276, 307-326.

Pierce, B.G., Hourai, Y., and Weng, Z. (2011). Accelerating protein docking in ZDOCK using an advanced $3 \mathrm{D}$ convolution library. PLOS One 6, e24657.

Recacha, R., Boulet, A., Jollivet, F., Monier, S., Houdusse, A., Goud, B., and Khan, A.R. (2009). Structural basis for recruitment of Rab6-interacting protein 1 to Golgi via a RUN domain. Structure (London, England: 1993) 17, 21-30.

Reichardt, L.F. (2006). Neurotrophin-regulated signalling pathways. Phi-los Trans R Soc Lond B Biol Sci 361, 1545-1564.

Rodrigueztebar, A., Dechant, G., and Barde, Y.A. (1990). Binding of brain-derived neurotrophic factor to the nerve growth-factor receptor. Neuron 4, 487-492.

Shooter, E.M. (2001). Early days of the nerve growth factor proteins. Annu Rev Neurosci 24, 601-629.

Vanhaesebroeck, B., Leevers, S.J., Ahmadi, K., Timms, J., Katso, R., Driscoll, P.C., Woscholski, R., Parker, P.J., and Waterfield, M.D. (2001). Synthesis and function of 3-phosphorylated inositol lipids. Annu Rev Biochem 70, 535-602.

Xing, J., Kornhauser, J.M., Xia, Z., Thiele, E.A., and Greenberg, M.E. (1998). Nerve growth factor activates extracellular 
signal-regulated kinase and p38 mitogen-activated protein kinase pathways to stimulate CREB serine 133 phosphorylation. Mol Cell Biol 18, 1946-1955.

Yang, H.B., Kim, O., Wu, J., and Qiu, Y. (2002). Interaction between tyrosine kinase Etk and a RUN domain- and FYVE domain-containing protein RUFY1 - A possible role of Etk in regulation of vesicle trafficking. J Biol Chem 277, 30219-30226.

Zhu, D.Y., Zhu, Y.Q., Xiang, Y., and Wang, D.C. (2005). Optimizing protein crystal growth through dynamic seeding. Acta Cryst D Biol Crystallogr 61, 772-775. 